\title{
X-Ray crystal structural determination of copper(II)-nitrilotriacetic acid-bis( $N$-methylimidazol-2-yl)ketone ternary complex
}

\author{
Christopher J. Burns,* Leslie D. Field, Trevor W. Hambley, Thomas Lin, Damon D. \\ Ridley, Peter Turner, and Matthew P. Wilkinson* \\ School of Chemistry and the Cooperative Research Centre for Molecular Engineering and \\ Technology, University of Sydney, Sydney N.S.W. 2006, Australia \\ E-mail: l.field@chem.usyd.edu.au
}

\section{Dedicated to Professor D. W. Cameron in appreciation of his significant contribution to Australian Chemistry \\ (received 30 Jul 01; accepted 19 Dec 01; published on the web 27 Dec 01)}

\begin{abstract}
The X-ray crystal structure of a ternary complex of copper(II)-nitrilotriacetic acid (NTA, 2) and bis( $N$-methylimidazol-2-yl)ketone $\mathbf{3}$ is reported. This structure provides information about the nature of the interaction of metal complexes of NTA with polyhistidine peptides.
\end{abstract}

Keywords: X-Ray crystal structure, ternary complex, copper (II)-nitriloacetic aicd, bis(Nmethylimidazole-2-yl)ketone

\section{Introduction}

Ternary metal complexes (metal complexes containing more than one type of ligand) have been widely studied for several decades due to their utility as model systems for metal-protein complexes observed in, for example, protein-protein interactions and present in certain proteins such as metalloenzymes. Transition metal ternary complexes have received particular focus and have been employed in mapping protein surfaces, ${ }^{1}$ as probes for biological redox centers, ${ }^{2}$ and in protein capture for both purification ${ }^{3}$ and study. ${ }^{4,5}$ The study of the structure of model ternary complexes provides information about how biological systems achieve their specificity and stability, as well as strategies to improve these features for biotechnological applications.

Ternary complexes of oxygen-donor ligands and heteroaromatic N-bases have attracted much interest as they can display exceptionally high stability. ${ }^{6,7,8}$ The use of transition metal complexes of iminodiacetic acid (IDA, 1) and nitrilotriacetic acid (NTA, 2) have been widely adopted in biology, and are gaining increasing use in biotechnology, particularly in the protein purification 
technique known as Immobilised Metal-ion Affinity Chromatography (IMAC). ${ }^{9}$ In IMAC, a metal binary complex (typically of $\mathrm{Cu}^{2+}, \mathrm{Ni}^{2+}$ or $\mathrm{Zn}^{2+}$ ) is covalently coupled to a permeable solid support such as agarose, and packed into a column. The protein to be purified is applied to the column, and selective binding of basic amino acids (particularly histidine) in the protein to the immobilised binary metal complex allows separation of histidine-rich proteins from other protein material. Proteins are now routinely engineered with a terminal polyhistidine sequence ("tag") using molecular biological techniques to allow purification by this method.

The first report of IMAC used IDA $\mathbf{1}$ as the covalently bound ligand to immobilise the metal ions to the solid support, ${ }^{10}$ and more recently NTA 2 has gained popularity as a metal chelator in IMAC. ${ }^{11}$ NTA 2 is a tetradentate ligand known to bind to a variety of transition metals with stability constants of $10^{9}$ to $10^{14},{ }^{12}$ and the use of NTA in IMAC is now commonplace with columns bearing immobilised NTA derivatives being commercially available.

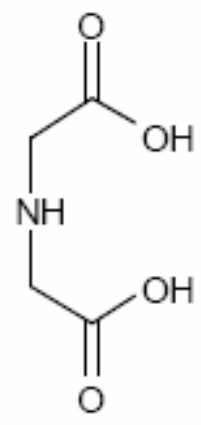

1

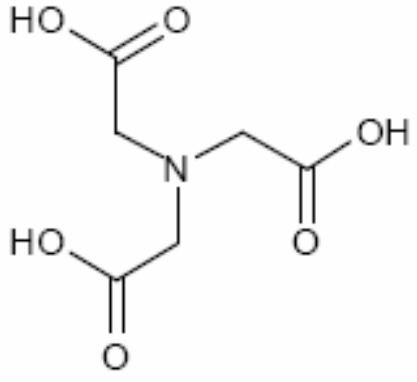

2

Since their initial use in IMAC, IDA-and NTA-metal chelates have been employed in a variety of applications including the crystallisation of proteins on monolayers, ${ }^{13}$ and in the temporary immobilization of proteins (particularly those possessing an engineered polyhistidine tag) on surfaces for biochemical study and imaging., ${ }^{4,5}$

Although the binding of proteins to tethered NTA and IDA metal complexes is being increasingly utilised, little structural information is known about the NTA- or IDAmetalpolyhistidine ternary complex fundamental to these processes. The interaction between a polyhistidine tag and a metal-NTA chelate is presumed to involve one nitrogen, three carboxylate ions and two histidines, based upon the steric and electronic requirements of the NTA system (Figure 1). 


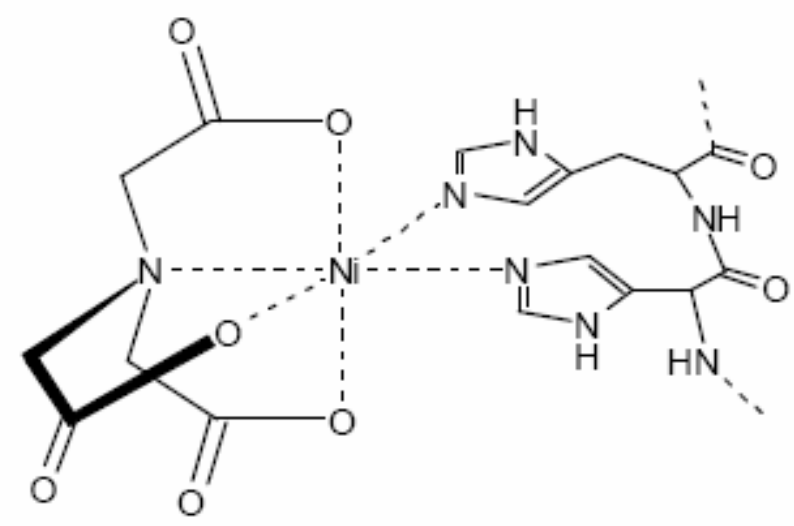

Figure 1. Proposed structure of NTA-metal-polyhistidine ternary complex.

While structural analysis of ternary complexes containing amino acids or peptides is difficult, ${ }^{14}$ single crystal X-ray diffraction has been used to structurally characterise a limited number of ternary metal complexes containing NTA and NTA derivatives. Only a few crystal structures of the general form M(NTA)(amino acid) however, have been reported: namely, Co.NTA.phenylalanine, ${ }^{15}$ Co.NTA.leucine, ${ }^{16}$ and Co.NTA.glycine. ${ }^{17}$ Furthermore, the crystal structure of Cr.NTA.Imidazole2 reported by Barton and coworkers, is the only reported crystal structure of a ternary complex containing an organic molecule analogous to the amino acid histidine. ${ }^{18}$ Chromium complexes of these types, however, are kinetically inert and not useful in IMAC. To our knowledge, there is no report of a crystal structure of a ternary complex containing NTA and a histidine analogue with the metal ions $\mathrm{Cu}^{2+}, \mathrm{Zn}^{2+}, \mathrm{Ni}^{2+}$ that are normally used in IMAC.

In an effort to improve our understanding of the interaction between NTA, metal ions used in IMAC, and histidine/polyhistidine sequences, simple bis-imidazole compounds were synthesised and their interaction with metal-NTA binary complexes was examined. Bis-imidazole compounds have been found to be useful as ligands for a wide range of transition metals in the field of organometallic catalysis. ${ }^{19}$ We reasoned that the bis-imidazole ketone $\mathbf{3}$ would be an excellent second ligand for a metal-NTA chelate, based on the coordination of this ligand in other species. Furthermore, the presence of two imidazole groups in close proximity is a good model for adjacent histidine moieties of a polyhistidine tag.

\section{Results and Discussion}

The bis-imidazole 3 was prepared following the literature method through lithiation of methyl imidazole and trapping the 2-lithio species with diethylcarbonate. ${ }^{20}$ The spectroscopic data obtained for the purified product were in full accord with the proposed structure and with the published data. A ternary complex of 3 with NTA and $\mathrm{Cu}^{2+}$ was prepared by mixing equimolar 
amounts of cupric nitrate, disodium NTA and $\mathbf{3}$ in water, and was recrystallised by slow diffusion of acetone into the aqueous solution at ambient temperature. Small blue crystals of the ternary complex were obtained and the X-ray crystal structure was determined (see experimental section for details).
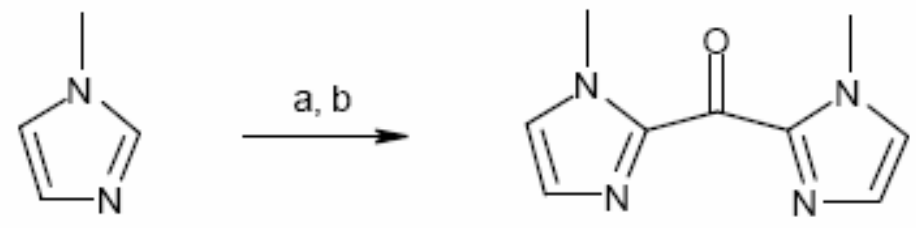

3

Scheme 1. (a) $n$-BuLi, THF, $-70 \nmid C, 1.5 \mathrm{~h}$; (b) (EtO) $2 \mathrm{CO}, \mathrm{THF},-70 \nmid \mathrm{C}, 1.5 \mathrm{~h}$.

An ORTEP projection of the molecule is shown in Figure 2, ${ }^{21}$ and several interesting features are immediately apparent. Firstly, only two of the three carboxylates in NTA are coordinating to the metal center and secondly, the copper has adopted a pyramidal shape (albeit slightly offset). Together, these observations are at odds with the accepted configuration of NTA and bisimidazole ligands about a metal ion normally assumed to give an octahedral complex (see Figure 1). An octahedral complex was also observed by Barton and coworkers for Cr.NTA.Imidazole2, ${ }^{18}$ however, as noted previously, $\mathrm{Cr}^{3+}$ is not used in techniques such as IMAC. As a consequence, the structure obtained in this present work may be closer to that normally adopted in ternary complexes involved in processes such as IMAC. Indeed, the fact that the third carboxylate of NTA is not bound to the metal is consistent with the similar efficacy observed for IDA species (where there is no third carboxylate) in IMAC and related applications. It should be noted however that in this model system the bis-imidazole is planar unlike the imidazole groups of a polyhistidine tag (which has many degrees of freedom) and therefore may hinder the complex from adopting an alternate structure. 


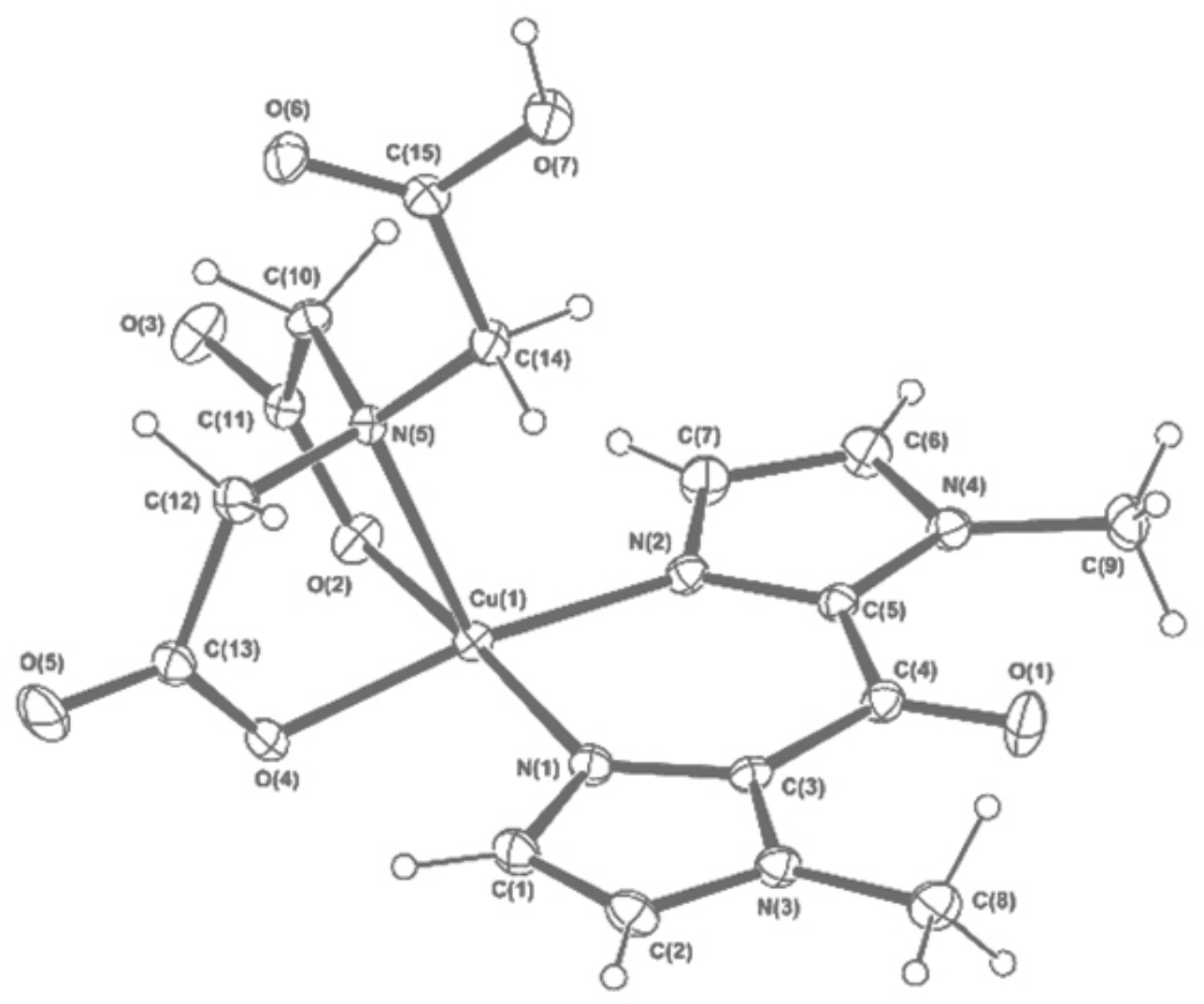

Figure 2. ORTEP drawing of crystal structure of bisimidazole 3 complexed to Cu(II) and NTA 2.

\section{Conclusions}

In this work the single crystal X-ray structure of a bis-imidazole ligand complexed to a $\mathrm{Cu}^{2+}$-NTA chelate has been obtained and indicates that the $\mathrm{Cu}$ species is in a pyramidal configuration, rather than the octahedral configuration generally proposed for a metal-NTA species complexed to two imidazole moieties. While this X-ray structure may not represent the structure of the complex in solution, and in addition is of a model system (where the rigidity of the bis-imidazole may be affecting the structure of the complex) this structure is possibly representative of NTA complexes present in techniques such as IMAC. Indeed, it is tempting to postulate that the free coordination site observed in this structure may normally be filled by a third imidazole moiety, from a third histidine in a polyhistidine tag. Tri-imidazole or related heterocyclic systems are therefore interesting species to test this hypothesis, and as new ligands for IDA-and NTA-metal chelates for applications in biotechnology. 


\section{Experimental Section}

General Procedures. All reactions were performed under an atmosphere of dry nitrogen. Anhydrous tetrahydrofuran was obtained by distillation from sodium benzophenone ketyl immediately prior to use. All other solvents were analytical grade and all reagents were commercial grade.

N.m.r. spectra were recorded on a Bruker AC-200F spectrometer (200 MHz for ${ }^{1} \mathrm{H} ; 50 \mathrm{MHz}$ for ${ }^{13} \mathrm{C}$ ) with samples dissolved in deuterated chloroform and referenced to solvent residual (7.26 ppm for ${ }^{1} \mathrm{H} ; 77.00 \mathrm{ppm}$ for ${ }^{13} \mathrm{C}$ ). Electron ionisation (e.i.) mass spectra were recorded on a modified Kratos mass spectrometer calibrated with perfluorokerosene (PFK). Microanalyses were performed by the Campbell Microanalytical Laboratory, Department of Chemistry, University of Otago, Dunedin, New Zealand.

Bis( $N$-methylimidazol-2-yl)ketone (3). Following the known procedure, freshly titrated $n$ butyllithium (45 mL, 1.7M, $76.5 \mathrm{mmol}$ ) was added dropwise to a solution of Nmethylimidazole (10 mL, $125 \mathrm{mmol})$ in tetrahydrofuran $(\sim 150 \mathrm{~mL})$ at $-70 \quad \mathrm{C}$ under nitrogen. The clear mixture was stirred for 1.5 hours and redistilled diethyl carbonate $(4.5 \mathrm{~mL}, 37.5 \mathrm{mmol})$ in tetrahydrofuran $(6.5 \mathrm{~mL})$ was added. The resulting pink mixture was maintained at $-70 \mathrm{C}$ for further 1.5 hours then warmed to $-40 \mathrm{C}$ and quenched by the addition of solid carbon dioxide. The reaction was allowed to warm to $10 \mathrm{C}$ followed by the addition of water $(20 \mathrm{~mL})$. This mixture was left overnight and filtered. The product was extracted from the organic filtrate with hydrochloric acid (4 $110 \mathrm{~mL}, 3 \mathrm{M}$ ). The combined aqueous layers were neutralised with sodium carbonate, filtered and the filtrate continuously extracted with chloroform in a liquid-liquid extractor for 16 hours. The organic layer obtained was dried $\left(\mathrm{MgSO}_{4}\right)$ and filtered through Celite ${ }^{\}}$. After removal of the solvent in vacuo, the product was recrystallised from hexane/dichloromethane to furnish bis $(N$ methylimidazol-2-yl)ketone 3 as pale yellow crystals (1.82g, 26\%), m.p. 151.2-153.0 C (lit. ${ }^{22}$ 147-148 C) (Found: $\mathrm{C}$, 56.8; $\mathrm{H}, 5.4 ; \mathrm{N}, 29.2 . \mathrm{C}_{9} \mathrm{H}_{10} \mathrm{~N}_{4} \mathrm{O}$ requires $\mathrm{C}, 56.8 ; \mathrm{H}, 5.3$; N, 29.5\%); ${ }^{1} \mathrm{H}-\mathrm{NMR} \delta 7.31$ (2H, s, 4-H), 7.09 (2H, s, 5-H), 4.01 (6H, s, N-CH $)$; ${ }^{13} \mathrm{C}-\mathrm{NMR} \delta 174.73(\mathrm{C}=\mathrm{O})$, 143.44 (C2), 130.82 (C4), 127.31 (C5), 36.42 ( $\left.\mathrm{CH}_{3}\right)$; m/z 190(M+4 47), 161(45), 109(100).

Preparation of $\mathrm{Cu}(\mathrm{NTA})$ (3). The bis-imidazole 3 (5 mg, $0.03 \mathrm{mmol}$ ), disodium nitrilotriacetic acid (6 mg, $0.03 \mathrm{mmol}$ ) and copper(II) nitrate $\bullet 3 \mathrm{H}_{2} \mathrm{O}(6 \mathrm{mg}, 0.03 \mathrm{mmol}$ ) were dissolved in distilled water $(0.5 \mathrm{~mL})$. The solution was placed into a container with an open top. This container was placed in another container containing acetone and the second container was sealed. Pale blue acicular crystals precipitated over 1 month. The solution was decanted to afford the copper ternary complex.

Crystal structure determination. A pale blue acicular crystal was attached to a thin glass fibre and mounted on a Rigaku AFC7R diffractometer employing graphite monochromated $\mathrm{CuK} \alpha$ radiation generated from a rotating anode. Cell constants were obtained from a least squares refinement against 25 reflections located between 70 and $91^{\circ} 2 \theta$. Data were collected at 295(2) 
Kelvin with $\omega-2 \theta$ scans to $135^{\circ} 2 \theta$. The intensities of 3 standard reflections measured every 150 reflections changed by 3.08 during the data collection and a correction was accordingly applied to the data. Lorentz and polarisation corrections were applied to the data, and an empirical absorption correction based on azimuthal scans of three suitable reflections was also applied to the data.

Processing and calculations were undertaken with TEXSAN. ${ }^{23}$ The structure was solved in the space group $P 2_{1} / \mathrm{c}(\# 14)$ by direct methods with SHELXS86, ${ }^{24}$ and extended and refined with SHELXL- $97^{25}$ using the TEXSAN interface. ${ }^{23}$ Anisotropic displacement parameters were refined for non-hydrogen atoms, and in general a riding atom model was used for the hydrogen atoms. The location of $\mathrm{H}(7 \mathrm{O})$ was identified and the atom was modeled with refined positional parameters and an isotropic displacement parameter.

There is a strong hydrogen bond between $\mathrm{O}(7)$ and $\mathrm{O}(3)$ on a neighbouring complex. An ORTEP $^{21,26}$ depiction of the molecule with $20 \%$ displacement parameters is provided in Figure 2. The crystal structure has been deposited at the Cambridge Crystallographic Data Centre and allocated the deposition number CCDC 167925.

\section{Acknowledgements}

We gratefully acknowledge financial support from the Australian Government through the Cooperative Research Centre for Molecular Engineering and Technology. 


\section{References and Notes}

1. Bocarsly, J. R.; Barton, J. K. Inorg. Chem. 1992, 31, 2827.

2. Farver, O.; Pecht, I.; Coord. Chem. Rev. 1989, 95, 17.

3. Crowe, J.; Döbeli, H.; Gentz, R.; Hochuli, E.; Stuber, D.; Henco, K. Methods Mol. Biol. 1994, 31, 371.

4. Nieba, L.; Nieba-Axmann, S. E.; Persson, A.; Hamalainen, M.; Edebratt, F.; Hansson, A.; Lidholm, J.; Magnusson, K.; Karlsson, A. F.; Pluckthun, A. Anal. Biochem. 1997, 252, 217.

5. Maloney, K. M.; Shnek, D. R.; Sasaki, D. Y.; Arnold, F. H. Chem. Biol. 1996, 3, 185.

6. Griesser, R.; Sigel, H. Inorg. Chem. 1970, 9, 1238.

7. Sigel, H. Inorg. Chem. 1980, 19, 1411.

8. Fischer, B. E.; Sigel, H. Inorg. Chem. 1979, 18, 425.

9. Arnold, F. H. Bio/Technology 1991, 9, 151.

10. Porath, J.; Carlsson, J.; Olsson, I.; Belfrage, G. Nature, 1975, 258, 598.

11. Hochuli, E.; Dobeli, H.; Schacher, A. J. Chromatog. 1987, 411, 177.

12. Anderegg, G. Pure Appl. Chem. 1982, 54, 2693.

13. Kubalek, E. W.; Le Grice, S. F. J.; Brown, P. O. J. Struct. Biol. 1994, 113, 117.

14. Martin, R. P.; Petit-Ramel, M. M.; Scharff, J. P. In Metal ions in biological systems, Marcel Dekker, 1976, Vol. 2, p. 1, (H. Sigel, ed.).

15. Hideyuki, K.; Koichiro, J.; Hideki, M.; Hisahiko, E. Inorg. Chim. Acta 1998, 283, 160.

16. Jitsukawa, K.; Morioka, T.; Masuda, H.; Ogoshi, H.; Einaga, H. Inorg. Chim. Acta 1994, 216, 249.

17. Gladkikh, O. P.; Polynova, T. N.; Porai-Koshits, M. A.; Poznyak. A. L. Koord. Khim. 1992, $18,1156$.

18. Bocarsly, J. R.; Chiang, M. Y.; Bryant, L.; Barton, J. K. Inorg. Chem. 1990, 29, 4898.

19. Burling, S.; Field, L. D.; Messerle, B. A. Organometallics 2000, 19, 87, and references therein.

20. Elgafi, S.; Field, L. D.; Messerle, B. A.; Turner, P.; Hambley, T. W. J. Organomet. Chem. 1999, 588, 69.

21. Johnson,C. K. ORTEPII: Report ORNL-5138, Oak Ridge National Laboratory, Oak Ridge, Tennessee, 1976.

22. Byers, P. K.; Canty, A. J. Organometallics 1990, 9, 210.

23. teXsan: Crystal Structure Analysis Package, Molecular Structure Corporation (1985 \& 1992).

24. Sheldrick, G. M. In Crystallographic Computing 3, Oxford University Press, 1985, pp. 175189, (Ed's G.M. Sheldrick, C. Kruger and R. Goddard).

25. Sheldrick, G. M. SHELXL97: Program for crystal structure refinement. University of Göttingen, Germany, 1997. 
26. Hall, S. R.; du Boulay, D. J.; Olthof-Hazekamp, R. In Xtal3.6 System, University of Western Australia, 1999. 